\title{
TRACE-POSITIVE NON-COMMUTATIVE POLYNOMIALS
}

\author{
RONAN QUAREZ
}

(Communicated by Harm Derksen)

\begin{abstract}
We give some examples of trace-positive non-commutative polynomials of degree 4 in 3 variables which are not cyclically equivalent to a sum of hermitian squares. Since some similar examples of degree 6 in 2 variables were alreay known, this settles a perfect analogy to Hilbert's result from the commutative context which says that positive (commutative) polynomials of degree $d$ in $n$ variables are not necessarily sums of squares, the first non-trivial cases being obtained for $(d, n)=(4,3)$ and $(d, n)=(6,2)$.
\end{abstract}

\section{INTRODUCTION}

Interest in the study of non-negative polynomials and sums of squares goes back to Hilbert and his famous 17th problem.

At the end of the 19th century, Hilbert showed Hi] that a polynomial $f \in$ $\mathbb{R}\left[x_{1}, \ldots, x_{n}\right]$ of even degree $d$ in $n$ variables over the reals which is non-negative on $\mathbb{R}^{n}$, is necessarily a sum of squares in $\mathbb{R}\left[x_{1}, \ldots, x_{n}\right]$ if and only if $n \leq 2$ or $d \leq 2$ or $(n, d)=(3,4)$.

One may reformulate this for forms (homogeneous polynomials), saying that positive forms are not necessarily sums of squares, the first non-trivial cases are obtained for $(n, d)=(3,6)$ and $(n, d)=(4,4)$. Suprisingly, explicit examples only appeared much later; the most celebrated one being the Motzkin polynomial [Mo]:

$$
m(x, y, z)=z^{6}+x^{2} y^{4}+y^{2} x^{4}-3 x^{2} y^{2} z^{2} .
$$

Other examples followed in the 1970s. For instance, due to CL, the following quaternary quartics is non-negative but not a sum of squares:

$$
q(x, y, z, w)=w^{4}+x^{2} y^{2}+x^{2} z^{2}+y^{2} z^{2}-4 x y z w .
$$

Our goal is to study the generalization of these notions when positivity is considered when evaluating at operators rather than real numbers. Namely, we may distinguish several different kinds of positivity that generalize the usual positivity of polynomials in commuting variables: namely matrix-positivity and trace-positivity.

A symmetric polynomial is matrix-positive if $F(A)$ is positive semidefinite for all $t$-uple of symmetric matrices of the same size. Note that for this evaluation to make sense we have to take $F$ in the $\mathbb{R}$-algebra of polynomials in non-commuting variables

Received by the editors May 14, 2013 and, in revised form, November 13, 2013 and January 9, 2014 .

2010 Mathematics Subject Classification. Primary 14P99, 15A63.

Key words and phrases. Positive semidefinite, sums of squares, sums of hermitian squares, trace-positivity.

The first author was supported by French National Research Agency (ANR) project GEOLMI - Geometry and Algebra of Linear Matrix Inequalities with Systems Control Applications. 
denoted by $\mathbb{R}\left\langle X_{1}, \ldots, X_{n}\right\rangle$. More precisely, $\mathbb{R}\langle X\rangle$ is the monoid ring of $\left\langle X_{1}, \ldots, X_{n}\right\rangle$ over $\mathbb{R}$ which is freely generated by the $n$ non-commuting letters $\left(X_{1}, \ldots, X_{n}\right)$.

Likewise, for trace-positivity: a polynomial $F \in \mathbb{R}\langle X\rangle$ is trace-positive if the trace of $F(A)$ is non-negative for all tuples $A$ of symmetric matrices of the same sizes.

These two notions of positivity for non-commutative polynomials are connected but they describe different sets of polynomials.

A result by $[\mathrm{He}$ says that a matrix-positive non-commutative polynomial $F$ is a sum of hermitian squares, which means that $F$ can be written as a non-negative linear combination of polynomials of the kind $P^{*} P$, where we endow $\mathbb{R}\langle X\rangle$ with the $\mathbb{R}$-algebra involution * that satisfies $\left(X_{i} X_{j}\right)^{*}=X_{j} X_{i}$. Note that this involution is compatible with the matrix transpose.

Concerning trace-positive polynomials, their investigation and the question of when they can be written as a sum of hermitian squares and commutators of polynomials is motivated by the connection to two famous conjectures: the BMV conjecture from statistical quantum mechanics and the embedding conjecture of Alain Connes concerning von Neumann algebras (see $\mathrm{Bu}$. for details).

In $[\mathrm{Bu}$, Theorem 1.11] the tracial analog of Hilbert's result on bivariate quartic polynomials has been proved. Namely: any bivariate trace-positive polynomial of degree at most four has such a representation as a sum of hermitian squares and commutators. This is false in general if the degree is at least six. One has some examples of polynomials which are trace-positive but are not a sum of hermitan squares and commutators. For instance the non-commutative version of the Motzkin polynomial is:

$$
M=1+X^{2} Y^{4}+Y^{2} X^{4}-3 X^{2} Y^{2} .
$$

This is in perfect analogy to Hilbert's results from the commutative context.

Note that there is still some difference with the commutative case. First, the polynomial $M$ is a non-homogeneous non-commutative version of $m$. Indeed, Hilbert's results about forms have a completely obvious equivalent version for (non-homogeneous) polynomials. But for non-commutative polynomials, even the homogenization operation is not well defined.

Second, there were no known examples of a trace-positive polynomial of degree 4 in 3 variables which is not a sum of hermitian squares and commutators.

We focus on this case, considering the following non-commutative dehomogenized version of $q$ :

$$
Q=1+X^{2} Y^{2}+X^{2} Z^{2}+Y^{2} Z^{2}-4 X Y Z
$$

Since it is not a sum of squares when we view it in the commutative world, it cannot be a sum of hermitian squares and commutators in $\mathbb{R}\langle X, Y, Z\rangle$. We prove in Theorem 3.4 that it is trace-positive by constructing an identity with denominators coming from commutative forms.

In the last section, we present a general process to obtain trace-positive polynomials (and also trace-positive forms) which are not sums of hermitian squares and commutators. But this process assumes a hypothesis known as the degree bounds conjecture for trace-positivity. Let us mention that this conjecture is related to the algebraic formulation of Connes's embedding conjecture as formulated in [KS]. 


\section{Preliminaries}

The ring of polynomials in $n$ commuting variables $x=\left(x_{1}, \ldots, x_{n}\right)$ is denoted by $\mathbb{R}[x]$. We denote by $\langle X\rangle$ the monoid which is freely generated by the $n$ noncommuting letters $X=\left(X_{1}, \ldots, X_{n}\right)$. Let $\mathbb{R}\langle X\rangle$ denote the monoid ring of $\langle X\rangle$ over $\mathbb{R}$. That is, the elements $F$ of $\mathbb{R}\langle X\rangle$ are polynomials in the non-commuting variables $X_{1}, \ldots, X_{n}$ with coefficients in $\mathbb{R}$, i.e. we may write non-commutative polynomials $F \in \mathbb{R}\langle X\rangle$ as

$$
F=\sum_{w \in\langle X\rangle} a_{w} w \in \mathbb{R}\langle X\rangle
$$

with $a_{w} \in \mathbb{R}$.

Let ${ }^{\wedge}: \mathbb{R}\langle X\rangle \rightarrow \mathbb{R}[x]$ be the algebra homomorphism mapping each $X_{i}$ to the commuting variable $x_{i}$. The image $\hat{F} \in \mathbb{R}[x]$ of a given polynomial $F \in \mathbb{R}\langle X\rangle$ is called the commutative collapse of $F$.

Instead of evaluating a polynomial $F \in \mathbb{R}\langle X\rangle$ on tuples of real numbers resulting in a real number we substitute $X$ by tuples $A=\left(A_{1}, \ldots, A_{n}\right)$ of symmetric matrices of same sizes. We endow $\mathbb{R}\langle X\rangle$ with the $\mathbb{R}$-algebra involution ${ }^{*}: \mathbb{R}\langle X\rangle \rightarrow \mathbb{R}\langle X\rangle$, $P \mapsto P^{*}$ that satisfies $\left(X_{i} X_{j}\right)^{*}=X_{j} X_{i}$. This involution is compatible with the matrix transpose, i.e. $F^{*}(A)=F(A)^{T}$ for all tuples $A$ of symmetric matrices of the same size. A polynomial $F \in \mathbb{R}\langle X\rangle$ is symmetric if $F^{*}=F$.

We say that $F$ is a sum of hermitian squares if $F$ can be written as a nonnegative linear combination of polynomials of the kind $P^{*} P$.

A polynomial $F \in \mathbb{R}\langle X\rangle$ is trace-positive if $\operatorname{Tr}(F(A)) \geq 0$ for all tuples $A$ of symmetric matrices of the same size, where $\operatorname{Tr}$ denotes the normalized trace of the canonical matricial trace $\operatorname{tr}$ (namely $\operatorname{Tr}(A)=\frac{\operatorname{tr}(A)}{d}$ when $A \in \mathbb{R}^{d \times d}$ ).

Of course, if $F$ is trace-positive, then $\hat{F}$ is positive. However the converse implication does not hold in general.

Since we are interested in the class of trace-positive polynomials, we endow the free algebra $\mathbb{R}\langle X\rangle$ with an equivalence relation to model the invariance of the trace under cyclic permutations. Namely, we say that two polynomials $F, G \in \mathbb{R}\langle X\rangle$ are cyclically equivalent $(F \sim G)$ if $F-G$ is a sum of commutators (elements of the form $[p, q]=p q-q p$ for $p, q \in \mathbb{R}\langle X\rangle)$ plus a sum of elements of the form $a_{w}\left(w-w^{*}\right)$ where $a_{w} \in \mathbb{R}$ and $w$ is a monomial. In other words, one has $F \sim G$ if and only if $\operatorname{tr}(f(\underline{A}))=\operatorname{tr}(g(\underline{A}))$ for any $n$-tuple $\underline{A}$ of symmetric matrices of the same size.

\section{A Positive SEMI-DEFinite FORM Which is NOT A SUM OF SQUARES}

Let us consider the following form in $\mathbb{R}[x]$ :

$$
q=w^{4}+x^{2} y^{2}+x^{2} z^{2}+y^{2} z^{2}-4 x y z w .
$$

An easy computation (see [CL]) shows that $q$ is not a sum of squares in $\mathbb{R}[x]$. Let us now use 4 different methods or algebraic certificates to show that $q$ is a positive form.

2.1. The arithmetico-geometric inequality. An easy application of the arithmetico-geometric inequality yields:

$$
\frac{1+x^{2} y^{2}+x^{2} z^{2}+y^{2} z^{2}}{4} \geq \sqrt[4]{x^{2} y^{2} x^{2} z^{2} y^{2} z^{2}}=x y z
$$


for $x \geq 0, y \geq 0, z \geq 0$. The other cases for the signs of $x, y, z$ are either obvious or reduce easily to this former case (up to multiplying some variables by -1 ).

2.2. Comparison to the unity. We have the identity:

$q=(x y-z)^{2}+(x z-y)^{2}-y^{2}-z^{2}+1+y^{2} z^{2}=(x y-z)^{2}+(x z-y)^{2}+\left(1-y^{2}\right)\left(1-z^{2}\right)$, which proves the positivity when $y^{2} \leq 1, z^{2} \leq 1$ or $y^{2} \geq 1, z^{2} \geq 1$. Note that this last condition is always satisfied up to permuting the variables $x, y, z$.

2.3. Substitution with odd powers. Another possibility would be to show that $q\left(x^{3}, y^{3}, z^{3}\right)$ is a sum of squares. The computer algebra system SOSTOOLS answers that this is the case. This device uses the powerfull techniques of interior points method to show the feasability of a semidefinite program which is the translation of our sum of squares problem. Unfortunately, it is not clear how to write down an explicit and "simple" sum of squares expression.

2.4. Identity with denominator. It is known form Artin's Theorem that $q$ is a sum of squares of rational functions. One may exhibit such an identity with a "simple" denominator:

$$
q(x, y, z)\left(1+x^{2}\right)=(1-x y z)^{2}+(y z-x)^{2}+\left(x^{2} y-x z\right)^{2}+\left(x^{2} z-x y\right)^{2},
$$

which obviously gives a certificate of positivity for $q$.

Let us see now if it is possible to extend to non-commutative liftings of $q$ some of the previous arguments of this section.

\section{Non-COMmutative LifTings}

In the non-commutative ring of freely generated polynomials $\mathbb{R}\langle X, Y, Z, W\rangle$, one may now consider the following lifting of the form $q$ :

$$
Q_{0}=W^{4}+X^{2} Y^{2}+X^{2} Z^{2}+Y^{2} Z^{2}-4 X Y Z W .
$$

It is not trace-positive if

$$
A=\left(\begin{array}{cc}
2 & -2 \\
-2 & 0
\end{array}\right) \quad B=\left(\begin{array}{cc}
-2 & -1 \\
-1 & 2
\end{array}\right) \quad C=\left(\begin{array}{cc}
-2 & -2 \\
-2 & -2
\end{array}\right) \quad D=\left(\begin{array}{cc}
2 & 0 \\
0 & -2
\end{array}\right),
$$

then

$$
\operatorname{Tr}\left(Q_{0}(A, B, C, D)\right)=-20 .
$$

Remark 3.1. Even the symmetrized version

$$
\begin{aligned}
& W^{4}+X^{2} Y^{2}+X^{2} Z^{2}+Y^{2} Z^{2} \\
& \quad-\frac{2}{3}(X Y Z W+X Y W Z+X Z Y W+X Z W Y+X W Y Z+X W Z Y)
\end{aligned}
$$

is not trace-positive.

That is why we will consider non-commutative liftings of the dehomogenized form $q$ by setting $w=1$. Let

$$
Q=1+X^{2} Y^{2}+X^{2} Z^{2}+Y^{2} Z^{2}-4 X Y Z .
$$

Note that we may also have considered the symmetrized version

$$
Q_{S}=1+X^{2} Y^{2}+X^{2} Z^{2}+Y^{2} Z^{2}-2(X Y Z+X Z Y),
$$

but it has same trace on triples of symmetric matrices since $\operatorname{Tr}(A B C)=\operatorname{Tr}(A C B)$ for all triple $(A, B, C)$ of symmetric matrices. 
Indeed, we have $Q \sim Q_{S}$ and hence $\operatorname{tr}(Q(A, B, C))=\operatorname{tr}\left(Q_{S}(A, B, C)\right)$ for all triple of symmetric matrices $(A, B, C)$.

Let us see how to study the trace-positivity of $Q$.

3.1. Arithmetico-geometric inequality. Since it is not an algebraic certificate, it seems that we cannot derive any non-trivial result about trace-positivity. The only thing which is clear is the trace-positivity of $Q$ evaluated on a triple of commuting matrices $(A, B, C)$. In that case $(A, B, C)$ are simultaneously diagonalizable and we are reduced to the commutative case.

3.2. Trace-positivity for contractions. The identity for commutative polynomials can be lifted in $\mathbb{R}\langle X, Y, Z\rangle$ to

$$
Q \sim(Y X-Z)^{*}(Y X-Z)+(X Z-Y)(X Z-Y)^{*}+\left(1-Y^{2}\right)\left(1-Z^{2}\right),
$$

which proves the trace-positivity when $Y^{2}-1, Z^{2}-1$ are both positive semi-definite or both negative semi-definite matrices. To see this, we recall the well known elementary result (we recall a proof for the convenience of the reader) which we will need also in the following:

Lemma 3.2. If $A$ and $B$ are two positive semi-definite symmetric matrices in $\mathbb{R}^{d \times d}$, then $\operatorname{tr}(A B) \geq 0$.

Proof. Since $A$ is positive semi-definite, it has a square root $C$, namely $A=C^{T} C$. Then, $\operatorname{tr}(A B)=\operatorname{tr}\left(C^{T} C B\right)=\operatorname{tr}\left(C B C^{T}\right) \geq 0$ since the matrix $C B C^{T}$ is positive semi-definite.

In particular, we get the trace-positivity of $Q$ when all eigenvalues of both $Y$ and $Z$ are in $[-1,1]$ ( $Y$ and $Z$ are both contractions) or are both not in $[-1,1]$.

3.3. Substitution with odd powers. Another possibility would be to show that $Q\left(X^{3}, Y^{3}, Z^{3}\right)$ is cyclically equivalent to a sum of hermitian squares. The commutative collapse of $Q\left(X^{3}, Y^{3}, Z^{3}\right)$ is surely a sum of squares as said, for instance, by the computer algebra system SOSTOOLS. Unfortunately, it is not clear how to obtain a "simple" identity that might be liftable in $\mathbb{R}\langle X, Y, Z\rangle$.

Another way to handle this would be to use the non-commutative version of a computer program dealing with hermitian squares. Such a device has been developed in CKP.

For instance, concerning the non-commutative Motzkin polynomial, such a certificate has been given by $\mathrm{K}$. Cafuta for the Motzkin polynomial $M$ (see $[\mathrm{Bu}]$ ), which gives another way of proving that it is trace-positive.

In our situation the computer program did not show if $Q\left(X^{3}, Y^{3}, Z^{3}\right)$ is cyclically equivalent to a sum of hermitian squares (due to the large number of monomials to handle: about 1093 in theory and more than 600 in practice!).

3.4. Identity with simple denominator. Remember the commutative identity that we previously considered:

$$
q(x, y, z)\left(1+x^{2}\right)=(1-x y z)^{2}+(y z-x)^{2}+\left(x^{2} y-x z\right)^{2}+\left(x^{2} z-x y\right)^{2} .
$$


Then, let us define the following non-commutative liftings:

$$
\left\{\begin{array}{l}
F_{1}=1-X Y Z \\
F_{2}=Y Z-X \\
F_{3}=X^{2} Y-X Z \\
F_{4}=X^{2} Z-X Y
\end{array}\right.
$$

and

$$
R=\sum_{i=1}^{4} F_{i}(X, Y, Z) F_{i}^{*}(X, Y, Z) .
$$

Let also $Q_{1}=1+Y Z^{2} Y+X Z^{2} X+X Y^{2} X-X Y Z-Z Y X-X Z Y-Y Z X$. Then,

$$
Q_{1}\left(1+X^{2}\right)=\sum_{i=1}^{4} F_{i} F_{i}^{*}+\sum_{i=1}^{5} U_{i}
$$

where

$$
\left\{\begin{array}{l}
U_{1}=X^{2} Z Y X-Z Y X^{3} \\
U_{2}=X^{2} Y Z X-Y Z X^{3} \\
U_{3}=Y Z^{2} Y X^{2}-X Y Z^{2} Y X \\
U_{4}=X Z^{2} X^{3}-X^{2} Z^{2} X^{2} \\
U_{5}=X Y^{2} X^{3}-X^{2} Y^{2} X^{2}
\end{array}\right.
$$

Before stating the result about our main example, we will need the following:

Lemma 3.3. Let $u_{1}=X^{a_{1}} v X^{b_{1}}$ and $u_{2}=X^{a_{2}} v X^{b_{2}}$ where $v \in \mathbb{R}\langle X, Y, Z\rangle$ and assume that $u_{1} \sim u_{2}$ (a condition that is equivalent to $a_{1}+b_{1}=a_{2}+b_{2}$ ).

Then, for all univariate polynomial $w \in \mathbb{R}\langle X\rangle$, we have $u_{1} w \sim u_{2} w$.

Proof. By linearity, it is enough to show that, for all $c \in \mathbb{N}$, we have $u_{1} X^{c} \sim u_{2} X^{c}$. This is obvious since $u_{i} X^{c} \sim v X^{a_{i}+b_{i}+c}$ for $i=1,2$.

We deduce the trace-positivity of $Q$ but also the locus where $\operatorname{Tr}(Q)$ vanishes:

Theorem 3.4. The polynomial $Q$ is trace-positive but not cyclically equivalent to a sum of hermitian squares. Moreover $\operatorname{Tr}(Q(A, B, C))=0$ if and only if $A=$ $\operatorname{Diag}\left(a_{i}\right)_{1 \leq i \leq d}, B=\operatorname{Diag}\left(b_{i}\right)_{1 \leq i \leq d}, C=\operatorname{Diag}\left(c_{i}\right)_{1 \leq i \leq d}$ where all the $a_{i}$ 's, $b_{i}$ 's and $c_{i}$ 's are in $\{-1,+1\}$ and such that $a_{i} b_{i} c_{i}=1$ for all $\bar{i}$.

Proof. The polynomial $Q$ is clearly not cyclically equivalent to a sum of hermitian squares since otherwise its commutative collapse and $q$ would be a sum of squares in $\mathbb{R}[x, y, z]$, which is not the case.

In equation (1), let us substitute $(X, Y, Z)$ by a triple of symmetric matrices $(A, B, C)$ in $\mathbb{R}^{d \times d}$. Then,

$$
Q_{1}(A, B, C)\left(\mathrm{Id}+A^{2}\right)=\sum_{i=1}^{4} F_{i}(A, B, C) F_{i}^{*}(A, B, C)+\sum_{i=1}^{5} U_{i}(A, B, C) .
$$

First notice that $\mathrm{Id}+A^{2}$ is invertible and $\left(\mathrm{Id}+A^{2}\right)^{-1}$ is a polynomial in $A$ which can be explicitely computed using the minimal or characteristic polynomial of $\operatorname{Id}+A^{2}$. Then, we get by Lemma 3.3 that

$$
\operatorname{Tr}\left(\sum_{i=1}^{5} U_{i}(A, B, C) \times\left(\mathrm{Id}+A^{2}\right)^{-1}\right)=0 .
$$


Hence,

(2) $\operatorname{Tr}\left(Q_{1}(A, B, C)\right)=\operatorname{Tr}\left(\left(\sum_{i=1}^{4} F_{i}(A, B, C) F_{i}^{*}(A, B, C)\right) \times\left(\mathrm{Id}+A^{2}\right)^{-1}\right)$

which is non-negative by Lemma 3.2 since $\sum_{i=1}^{4} F_{i}(A, B, C) F_{i}^{*}(A, B, C)$ is positive semi-definite and $\left(\mathrm{Id}+A^{2}\right)^{-1}$ is positive definite.

This shows the trace-positivity of $Q$ since $\operatorname{Tr}(Q(A, B, C))=\operatorname{Tr}\left(Q_{1}(A, B, C)\right)$ for all triple $(A, B, C)$ of symmetric matrices (although $Q$ and $Q_{1}$ are not cyclically equivalent).

The identity (2) also gives the locus where $\operatorname{Tr}(Q)$ vanishes. Indeed, if $\operatorname{Tr}(Q(A, B, C))=0$, then for all $i, \operatorname{Tr}\left(F_{i}(A, B, C) F_{i}^{*}(A, B, C)\right)=0$ which means that $F_{i}(A, B, C)=0$. From this, one easily deduces that $A, B, C$ are invertible and commute. The result follows.

Remark 3.5. In the previous proof it is crucial that the inverse of a given matrix $M$ is a polynomial in $M$, which implies that our argument does not generalize to infinite objects like von Neumann algebras.

\section{Some OTHER EXAMPLES}

We may use the techniques given in Section 3.4 to produce other examples of polynomials that are trace-positive but not a sum of hermitian squares.

4.1. Another dehomogenization of $Q_{0}$. We choose now to dehomogenize $Q_{0}$ by setting $X=1$ :

$$
\widetilde{Q}(Y, Z, W)=W^{4}+Y^{2}+Z^{2}+Y^{2} Z^{2}-4 Y Z W .
$$

Let us define the non-commutative liftings:

$$
\left\{\begin{array}{l}
G_{1}=W^{3}-Y Z, \\
G_{2}=W Y Z-W^{2}, \\
G_{3}=Y-W Z, \\
G_{4}=Z-W Y .
\end{array}\right.
$$

and also $S=\sum_{i=1}^{4} G_{i}(Y, Z, W) G_{i}^{*}(Y, Z, W)$.

Let us also define $Q_{2}=W^{4}+Y^{2}+Z^{2}+Y Z^{2} Y-2(Y Z W+Z Y W)$. Then,

$$
Q_{2}\left(1+W^{2}\right)=S+\sum_{i=1}^{7} V_{i}
$$

where

$$
\left\{\begin{array}{l}
V_{1}=-Y Z W+W Y Z \\
V_{2}=-Z Y W+W Z Y \\
V_{3}=-Y Z W^{3}+W Y Z W^{2} \\
V_{4}=-2 Z Y W^{3}+W^{3} Z Y+W^{2} Z Y W \\
V_{5}=Y^{2} W^{2}-W Y^{2} W \\
V_{6}=Z^{2} W^{2}-W Z^{2} W \\
V_{7}=Y Z^{2} Y W^{2}-W Y Z^{2} Y W
\end{array}\right.
$$

Proposition 4.1. The polynomial $\widetilde{Q}$ is trace-positive but not cyclically equivalent to a sum of hermitian squares. 
Proof. We proceed as in the proof of Theorem 3.4.

Since $\left(\operatorname{Id}+D^{2}\right)^{-1}$ is a polynomial in $D$, we get by Lemma 3.3 that,

$$
\operatorname{Tr}\left(\sum_{i=1}^{6} V_{i}(A, B, C) \times\left(\mathrm{Id}+D^{2}\right)^{-1}\right)=0 .
$$

Hence,

$$
\operatorname{Tr}\left(Q_{2}(B, C, D)\right)=\operatorname{Tr}\left(\left(\sum_{i=1}^{4} G_{i}(B, C, D) G_{i}^{*}(B, C, D)\right) \times\left(\operatorname{Id}+D^{2}\right)^{-1}\right),
$$

which is non-negative since $\sum_{i=1}^{4} G_{i}(B, C, D) G_{i}^{*}(B, C, D)$ is positive semi-definite and $\left(\operatorname{Id}+D^{2}\right)^{-1}$ is positive definite.

This concludes the proof since and $\operatorname{Tr}(\widetilde{Q}(A, B, C))=\operatorname{Tr}\left(Q_{2}(A, B, C)\right)$ for all triple $(A, B, C)$ of symmetric matrices.

For convenience, one may introduce the following notation for $u \in \mathbb{R}\langle X, Y, Z, W\rangle$ :

$$
\mathcal{C}_{u}^{0}=\left\{v \in \mathbb{R}\langle X, Y, Z, W\rangle \mid \forall k \in \mathbb{N}, v u^{k} \sim 0\right\} .
$$

With this notation, Lemma 3.3 says that $X^{a_{1}} v X^{b_{1}}-X^{a_{2}} v X^{b_{2}} \in \mathcal{C}_{X}^{0}$ where $v \in$ $\mathbb{R}\langle X, Y, Z\rangle$ and $a_{1}+b_{1}=a_{2}+b_{2}$.

4.2. The Motzkin polonymial. It is already known that

$$
M=1+X^{2} Y^{4}+Y^{2} X^{4}-3 X^{2} Y^{2} .
$$

is trace-positive but not cyclically equivalent to a sum of hermitian squares. See $\mathrm{Bu}$ for several different proofs.

Proceeding as in section 3.4, one can give an alternate algebraic certificate to show that $M$ is trace-positive. Indeed, we start with the commutative identity

$$
\left(1+x^{2} y^{4}+y^{2} x^{4}-3 x^{2} y^{2}\right)\left(1+x^{2}\right)=\left(1-x^{2} y^{2}\right)^{2}+\left(x^{3} y-x y\right)^{2}+\left(x y^{2}-x\right)^{2} .
$$

and one can show that it is possible to derive a non-commutative identity of the form

$$
\begin{aligned}
M(1 & \left.+X^{2}\right)-\left(\left(1-X^{2} Y^{2}\right)\left(1-X^{2} Y^{2}\right)^{*}\right. \\
& \left.+\left(X^{3} Y-X Y\right)\left(X^{3} Y-X Y\right)^{*}+\left(X Y^{2}-X\right)\left(X Y^{2}-X\right)^{*}\right) \in \mathcal{C}_{X}^{0} .
\end{aligned}
$$

Likewise, one can show that

$$
\widetilde{M}=Z^{6}+Y^{4}+Y^{2}-3 Y^{2} Z^{2}
$$

is trace-positive by giving an algebraic certificate of the form $\widetilde{M}\left(1+Z^{2}\right)-\left(\left(Z^{4}-Y^{2}\right)\left(Z^{4}-Y^{2}\right)^{*}+\left(Y-Z^{2} Y\right)\left(Y-Z^{2} Y\right)^{*}+\left(Z Y^{2}-Z^{3}\right)\left(Z Y^{2}-Z^{3}\right)^{*}\right) \in \mathcal{C}_{Z}^{0}$.

Using the same proof as in the previous examples, one may write a tracepositivity certificate with denominator:

Theorem 4.2. Let $P \in \mathbb{R}\langle\underline{X}\rangle$ where $\underline{X}=\left(X_{1}, \ldots, X_{n}\right)$. Assume that there are some some of hermitian squares $Q_{1}, Q_{2}$ in $\mathbb{R}\langle\underline{X}\rangle$ and $R \in \mathcal{C}_{Q_{1}}^{0} \subset \mathbb{R}\langle\underline{X}\rangle$ such that

$$
\left(1+Q_{1}\right) P=Q_{2}+R .
$$

Then, the polynomial $P$ is trace-positive. 


\section{TOWARDS SOME NON-COMMUTATIVE HOMOGENEOUS EXAMPLES}

5.1. Degree bounds conjecture. One may want to construct examples of (homogeneous) non-commutative forms which are trace-positive but not cyclically equivalent to a sum of hermitian squares. We will see that this is possible when assuming the so-called degree bounds conjecture. The latter can be stated as follows:

Conjecture 5.1 (Degree bounds). For any integer $g$, there is an integer $d=d(g, n)$ such that the following holds: any polynomial $P \in \mathbb{R}\left\langle X_{1}, \ldots, X_{n}\right\rangle$ of degree $g$ is trace-positive if and only if $\operatorname{Tr}\left(P\left(A_{1}, \ldots, A_{n}\right)\right) \geq 0$ for all symmetric matrices $A_{1}, \ldots, A_{n}$ in $\mathbb{R}^{d \times d}$.

First of all, note that the counterpart degree bounds property in the context of matrix positivity holds true (by $[\mathrm{He}$ ).

Conjecture 5.1 is, in some sense, related to Connes's embedding conjecture although we deal here with the normalized Hilbert-Schmidt norm $\|M\|=\sqrt{\operatorname{Tr}\left(M^{T} M\right)}$ instead of the operator norm $\|M\|_{\text {op }}$ which is equal to the supremum of the eigenvalues of $M^{T} M$. Indeed, to see the analogy, let us first formulate an analogous degree bounds conjecture on the hypercube:

Conjecture 5.2 (Degree bounds on the hypercube). For any integer $g$, there is an integer $d=d(g, n)$ such that the following holds: any polynomial $P \in$ $\mathbb{R}\left\langle X_{1}, \ldots, X_{n}\right\rangle$ of degree $g$ is trace-positive on all $n$-tuples $\left(A_{1}, \ldots, A_{n}\right)$ of symmetric operators lying in the Hilbert-Schmidt hypercube (i.e. for all $i, \operatorname{Tr}\left(A_{i} A_{i}^{T}\right) \leq 1$ and $\left.A_{i}=A_{i}^{T}\right)$ if and only if $\operatorname{Tr}\left(P\left(A_{1}, \ldots, A_{n}\right)\right) \geq 0$ for all $n$-tuples of symmetric operators in $\mathbb{R}^{d \times d}$ lying in the Hilbert-Schmidt hypercube.

Let us remark that Conjecture 5.2 implies Conjecture 5.1

Since operators having operator norm less than or equal to 1 are just contractions, one can see the connection with the algebraic formulation of Connes's embedding conjecture as formulated in $[\mathrm{KS}]$ :

Conjecture (Conne's embedding). Any polynomial $P \in \mathbb{R}\left\langle X_{1}, \ldots, X_{n}\right\rangle$ of degree $g$ is trace-positive on all $n$-tuple $\left(A_{1}, \ldots, A_{n}\right)$ of symmetric contractions if and only if $P$ is trace-positive on all $\mathrm{II}_{1}$-factors.

We return now to our degree bounds Conjecture 5.1, Let us now mention some elementary known cases when this is true:

(1) When $g=2$, we have $d(2, n)=1$. Moreover in that case a trace-positive polynomial is cyclically equivalent to a sum of hermitian squares.

(2) When $g=4$ and $n=2$ we have $d(4,2)=2$ (see [BK]). Moreover, in that case a trace-positive polynomial is cyclically equivalent to a sum of hermitian squares.

Furthermore, if a polynomial is cyclically sorted in 2 variables, it is trace-positive if and only if its commutative collapse is positive. Namely it is enough to check trace-positivity for matrices of size 1 .

Moreover, because of the polynomial identities $[\mathrm{Rw}$, one has some lower bound for $d(g, n)$. For instance, there exists a polynomial of degree 4 in 4 variables which vanishes on any 4 -tuple of symmetric matrices in $\mathbb{R}^{2 \times 2}$. Hence, even for quaternary quartics, it is necessary to check trace-positivity at least on $3 \times 3$ matrices. In other words $d(4,4) \geq 3$. 
Assuming the degree bounds conjecture, let us see how to construct some examples of trace-positive forms which are not sums of hermitian squares.

For $\eta \geq 0$, let us define the (commutative) form

$$
q_{\eta}=q+\eta\left(x^{2}+y^{2}+z^{2}\right)=w^{4}+x^{2} y^{2}+x^{2} z^{2}+y^{2} z^{2}-4 x y z w+\eta\left(x^{2}+y^{2}+z^{2}\right) .
$$

We need an identity about commutative forms coming from $\mathrm{Ve}$, (1.13) and section $3]$ :

Proposition 5.3. The form $q_{\eta}$ is positive-semi-definite but not a sum of squares for any $\eta$ such that $0 \leq \eta<\eta_{0}$ where $\sqrt{\eta_{0}}$ is the smallest positive root of $s^{3}-\frac{1}{2} s+\frac{1}{9}=0$ $\left(\sqrt{\eta_{0}} \sim 0.25\right)$. Further,

$$
\begin{aligned}
q_{\eta_{0}}=\left(w^{2}-\sqrt{\eta_{0}}\left(x^{2}+y^{2}+z^{2}\right)\right)^{2}+ & \frac{2}{9 \sqrt{\eta_{0}}}\left(\left(3 \sqrt{\eta_{0}} w x-y z\right)^{2}\right. \\
& \left.+\left(3 \sqrt{\eta_{0}} w y-z x\right)^{2}+\left(3 \sqrt{\eta_{0}} w z-x y\right)^{2}\right) .
\end{aligned}
$$

Let us consider the following non-commutative liftings:

$$
\begin{array}{lll}
H_{0}=W^{2}-\sqrt{\eta_{0}}\left(X^{2}+Y^{2}+Z^{2}\right) & \\
H_{1}=3 \sqrt{\eta_{0}} W X-Y Z & K_{1}=3 \sqrt{\eta_{0}} X W-Y Z \\
H_{2}=3 \sqrt{\eta_{0}} W Y-X Z & K_{2}=3 \sqrt{\eta_{0}} Y W-X Z \\
H_{3}=3 \sqrt{\eta_{0}} W Z-X Y & K_{3}=3 \sqrt{\eta_{0}} Z W-X Y
\end{array}
$$

and

(3) $Q_{\eta_{0}}=H_{0}^{*} H_{0}+\frac{1}{9 \sqrt{\eta_{0}}}\left(H_{1}^{*} H_{1}+K_{1}^{*} K_{1}+H_{2}^{*} H_{2}+K_{2}^{*} K_{2}+H_{3}^{*} H_{3}+K_{3}^{*} K_{3}\right)$

and finally

$$
Q_{\eta}=Q_{\eta_{0}}+\left(\eta-\eta_{0}\right)\left(X^{4}+Y^{4}+Z^{4}\right) .
$$

Remark that $Q_{\eta}$ is also cyclically equivalent to the following polynomial:

$$
\begin{aligned}
& Q_{\eta} \sim W^{4}+\eta\left(X^{4}+Y^{4}+Z^{4}\right)+\left(2 \eta_{0}+\frac{2}{9 \sqrt{\eta_{0}}}\right)\left(X^{2} Y^{2}+X^{2} Z^{2}+Y^{2} Z^{2}\right) \\
& -\frac{2}{3}(X Y Z W+X Y W Z+X Z Y W+X Z W Y+X W Y Z+X W Z Y) .
\end{aligned}
$$

We have:

Proposition 5.4. Let us assume that the degree bounds Conjecture 5.1 holds true for polynomials of degree 4 in 4 variables.

There is then an $\eta \in] 0, \eta_{0}\left[\right.$ such that $Q_{\eta}$ is trace-positive but not a sum of hermitian squares.

Proof. Following the notations of subsection 5.1 let us set $d=d(4,4)$.

Since $Q_{\eta_{0}}$ is trace-positive (it is a sum of hermitian squares), it is trace-positive on matrices of size $d$.

First of all, note that the only roots of $Q_{\eta_{0}}$ are trivial. Indeed, let $(A, B, C, D)$ be a quadruple of symmetric matrices in $\mathbb{R}^{d \times d}$ which is a root of $Q_{\eta_{0}}$. Since $H_{i}(A, B, C, D)=0$ and $K_{i}(A, B, C, D)=0$ for all $i=1,2,3$, the matrices $A, B$, $C, W$ all pairwise commute; they are simultaneously diagonalizable. Since $q_{\eta_{0}}$ has only trivial roots, we deduce that $A=B=C=D=0$.

Let

$$
S^{d}=\left\{(A, B, C, D) \in\left(\mathcal{S} \mathbb{R}^{d \times d}\right)^{4} \mid \operatorname{Tr}\left(A^{4}+B^{4}+C^{4}+D^{4}\right)=1\right\},
$$

where $\mathcal{S} \mathbb{R}^{d \times d}$ denotes the set of all symmetric matrices in $\mathbb{R}^{d \times d}$. 
The set $S^{d}$ is a compact set, and since $\operatorname{Tr}\left(Q_{\eta_{0}}\right)$ does not vanish, we have on $S^{d}$

$$
\operatorname{Tr}\left(Q_{\eta_{0}}\right) \geq \epsilon>0 \text {. }
$$

Thus, for all $(A, B, C, D) \in S^{d}$ :

$$
\operatorname{Tr}\left(Q_{\eta_{0}}(A, B, C, D)\right)-\epsilon \operatorname{Tr}\left(A^{4}+B^{4}+C^{4}+D^{4}\right) \geq 0,
$$

and hence for all $(A, B, C, D) \in \mathcal{S} \mathbb{R}^{d \times d}$

$$
\operatorname{Tr}\left(Q_{\eta_{0}}(A, B, C, D)-\epsilon\left(A^{4}+B^{4}+C^{4}\right)\right) \geq 0 .
$$

In other words $Q_{\eta_{0}-\epsilon}(X, Y, Z, W)$, which is homogeneous and trace-positive on $S^{d}$, is trace-positive since we have assumed the degree bounds conjecture. Furthermore, it is not cyclically equivalent to a sum of hermitian squares since $q_{\eta_{0}-\epsilon}$ is not a sum of squares because of Proposition 5.3 .

Remark 5.5. Let us define the sequence of real numbers $m_{k}=\inf _{S^{k}} \operatorname{Tr}\left(Q_{\eta_{0}}\right)$. Then, the decreasing sequence $\left(m_{k}\right)_{k \in \mathbb{N}}$ is stationary if we have the degree bounds conjecture. By contraposition, if the sequence $\left(m_{k}\right)_{k \in \mathbb{N}}$ is not stationary, then it would give a counterexample to the degree bounds conjecture (for the trace-positivity of $\left.Q_{\eta_{0}}\right)$.

5.2. General procedure. Let $p$ be a commutative form of degree $2 d$ in the variables $x_{1}, \ldots, x_{n}$. Assume that $p$ is positive definite but not a sum of squares in $\mathbb{R}\left[x_{1}, \ldots, x_{n}\right]$.

A result by Robinson ([Ro]) says that for a big enough real number $\eta$, the form $p+\eta\left(x_{1}^{2 d}+\ldots+x_{n}^{2 d}\right)$ is a sum of squares. Hence,

$$
q_{\eta}=p+\eta\left(\sum_{1 \leq i \leq n} x_{i}^{2 d}+\sum_{1 \leq i, j \leq n} x_{i}^{2 d-2} x_{j}^{2}\right)
$$

is a sum of squares in $\mathbb{R}\left[x_{1}, \ldots, x_{n}\right]$. Let us consider the smallest real number $\eta_{0}$ such that $q_{\eta_{0}}$ is a sum of squares. Let us write

$$
q_{\eta_{0}}=\sum_{i=1}^{r} f_{i}^{2} .
$$

There is some $f_{k}$ which can be written as

$$
f_{k}=a_{k} x_{1}^{d}+b_{k} x_{1}^{d-2} x_{2}^{2}+g_{k}
$$

or

$$
f_{k}=c_{k} x_{1}^{d-1} x_{2}+h_{k}
$$

where $a_{k}, b_{k}, c_{k}$ are non-zero real numbers and the monomials in $g_{k}$ and $h_{k}$ have smaller lexicographic ordering than $x_{1}^{d-2} x_{2}^{2}$ or $x_{1}^{d-1} x_{2}$ respectively.

Let us consider the non-commutative liftings:

or

$$
\left\{\begin{array}{l}
F_{k, 1}=a_{k} X_{1}^{d}+b_{k} X_{1}^{d-2} X_{2}^{2}+G_{k} \\
F_{k, 2}=a_{k} X_{1}^{d}+b_{k} X_{2}^{2} X_{1}^{d-2}+G_{k}
\end{array}\right.
$$

$$
\left\{\begin{array}{l}
F_{k, 1}=c_{k} X_{1}^{d-1} X_{2}+H_{k} \\
F_{k, 2}=c_{k} X_{2} X_{1}^{d-1}+H_{k}
\end{array}\right.
$$

where $G_{k}$ and $H_{k}$ are arbitrary liftings of $g_{k}$ and $h_{k}$.

We then repeat the same lifting procedure for any couple of indexes $(i, j)$ instead of $(1,2)$. 
After a suitable averaging, we get an identity

$$
P_{\eta_{0}}=\sum_{k=1}^{s} F_{k} F_{k}^{*} \sim P+\eta_{0}\left(\sum_{1 \leq i \leq n} X_{i}^{2 d}+\sum_{1 \leq i, j \leq n} X_{i}^{2 d-2} X_{j}^{2}\right),
$$

where $P$ is a non-commutative lifting of $p$.

The argument in the proof of Proposition 5.4 follows readily. Indeed, the equalities $F_{k, 1}\left(A_{1}, \ldots, A_{n}\right)=F_{k, 2}\left(A_{1}, \ldots, A_{n}\right)=0$ imply that $A_{1}^{d-2}$ and $A_{2}^{2}$ commute. Hence $A_{1}$ and $A_{2}$ commute. The same holds for any couple of matrices. We therefore get that $P_{\eta_{0}}$ does not vanish on $S^{d}$ since the form $p$ is positive definite.

Hence, assuming the degree bounds conjecture, one may conclude that there exists some $0<\eta<\eta_{0}$ such that $P_{\eta}$ is trace-positive but not cyclically equivalent to a sum of hermitian squares.

5.3. Uniform approximation of operators. To avoid the use of Conjecture 5.1. one may try to proceed by approximation with respect to the Hilbert-Schmidt norm.

Roughly speaking, the idea is the following: if $\operatorname{Tr}\left(Q_{\eta_{0}}(A, B, C, D)\right)$ were very small for a quadruple of symmetric matrices in $S^{d}$ then, by equation (3) and the triangular inequality, we would deduce that all the commutators $[A, B],[A, C]$ and $[B, C]$ are small. We would then approximate the quadruple $(A, B, C, D)$ by a pairwise commuting quadruple $(\widetilde{A}, \widetilde{B}, \widetilde{C}, \widetilde{D})$. Noticing that $q_{\eta_{0}}$ never vanishes, we would have

$$
\operatorname{Tr}\left(Q_{\eta_{0}}(\widetilde{A}, \widetilde{B}, \widetilde{C}, \widetilde{D})\right) \geq m>0
$$

where

$$
m=\inf _{\left\{x^{4}+y^{4}+z^{4}+w^{4}=1\right\}} q_{\eta_{0}}(x, y, z, w) .
$$

If $\operatorname{Tr}\left(Q_{\eta_{0}}(A, B, C, D)\right)$ were close enough to $\operatorname{Tr}\left(Q_{\eta_{0}}(\widetilde{A}, \widetilde{B}, \widetilde{C}, \widetilde{D})\right)$, by approximation, we would get a contradiction showing that $\operatorname{Tr}\left(Q_{\eta_{0}}\right)$ is bounded from below in $S^{d}$.

The main problem we face is that we need some uniformity with respect to the dimension $d$.

There is a deep approximation result by Lin [L] with respect to the operator norm $\|\cdot\|_{\text {op }}$.

In the same spirit, but maybe more appropriate for our purpose on tracepositivity, there is a recent result by Glebsky Gy dealing with normalized HilbertSchmidt norm:

Theorem 5.6 (Glebsky). Let $\delta>0$. For any $k \in \mathbb{N}$, there is an $\epsilon=\epsilon(\delta, k)$ such that if $\left\|\left[A_{i}, A_{j}\right]\right\| \leq \epsilon$ for symmetric matrices $A_{1}, \ldots, A_{k}$ in $\mathbb{R}^{d \times d}$ with $\left\|A_{i}\right\|_{\text {op }} \leq 1$, then there exist parwise commuting symmetric matrices $B_{1}, \ldots, B_{k}$ in $\mathbb{R}^{d \times d}$ such that $\left\|B_{j}-A_{j}\right\| \leq \delta$ and $\left\|B_{i}\right\|_{\text {op }} \leq 1$.

Unfortunately, we did not use this result because of the assumption (natural but not convenient for our example) that the operators should be bounded for the operator norm. For the moment, we only have the following partial result:

Proposition 5.7. For any positive real number $M$, there exists $\eta>0$ such that $Q_{\eta}$ is non-cyclically equivalent to a sum of hermitian squares but that is trace-positive on any quadruple of symmetric operators whose operator norm is bounded by $M$. 
Proof. Let

$$
\begin{gathered}
S_{1, M}^{d}=\left\{(A, B, C, D) \in S \mathbb{R}^{d \times d} \mid\|A\|_{\text {op }} \leq M,\|B\|_{\text {op }} \leq M,\|C\|_{\text {op }} \leq M,\|D\|_{\text {op }} \leq M\right. \\
\left.\left\|X^{2}\right\|^{2}+\left\|Y^{2}\right\|^{2}+\left\|Z^{2}\right\|^{2}+\left\|W^{2}\right\|^{2}=1\right\} .
\end{gathered}
$$

Note that $\operatorname{Tr}\left(A^{4}+B^{4}+C^{4}+D^{4}\right)=\left\|A^{2}\right\|^{2}+\left\|B^{2}\right\|^{2}+\left\|C^{2}\right\|^{2}+\left\|D^{2}\right\|^{2}$.

Let

$$
\begin{gathered}
B_{M, 3 / 2}^{d}=\left\{(A, B, C, D) \in S \mathbb{R}^{d \times d} \mid\|A\|_{\text {op }} \leq M,\|B\|_{\text {op }} \leq M,\|C\|_{\text {op }} \leq M,\|D\|_{\text {op }} \leq M\right. \\
\left.1 / 2 \leq \operatorname{Tr}\left(A^{4}+B^{4}+C^{4}+D^{4}\right) \leq 3 / 2\right\} .
\end{gathered}
$$

We argue by contradiction. Assume that, for all $\epsilon>0$, there are $d$ and $(A, B, C, D)$ $\in S_{1, M}^{d}$, such that

$$
\operatorname{Tr}\left(Q_{\eta_{0}}(A, B, C, D)\right)<\epsilon
$$

Then,

$$
\frac{1}{9 \sqrt{\eta_{0}}} \operatorname{Tr}\left(F_{i}^{*} F_{i}\right)(A, B, C, D)<\epsilon
$$

and

for all $i=1,2,3$.

$$
\frac{1}{9 \sqrt{\eta_{0}}} \operatorname{Tr}\left(G_{i}^{*} G_{i}\right)(A, B, C, D)<\epsilon
$$

The triangular inequality gives, up to resizing $\epsilon$ :

$$
\|[B, C]\|=\|B C-C B\| \leq\left\|B C-3 \sqrt{\eta_{0}} D A\right\|+\left\|3 \sqrt{\eta_{0}} D A-C B\right\|<2 \epsilon
$$

and a similar inequality holds for the other commutators.

Since we have assumed that $A, B, C, D \in S_{1, M}^{d}$ have operator norms less than $M$, we may use Theorem 5.6, there are pairwise commuting $A^{\prime}, B^{\prime}, C^{\prime}, D^{\prime}$ whose operator norms are less than $M$ such that

$$
\left\{\begin{array}{l}
\left\|A^{\prime}-A\right\| \leq \sqrt{M} \delta \\
\left\|B^{\prime}-B\right\| \leq \sqrt{M} \delta \\
\left\|C^{\prime}-C\right\| \leq \sqrt{M} \delta \\
\left\|D^{\prime}-D\right\| \leq \sqrt{M} \delta .
\end{array}\right.
$$

In particular, we may assume that $A^{\prime}, B^{\prime}, C^{\prime}, D^{\prime} \in B_{3 / 2}^{d}$ up to resizing $\delta$.

We have uniform continuity of $\operatorname{Tr}\left(Q_{\eta_{0}}\right)$ on the compact set $B_{3 / 2, M}^{d}$ with respect to the Hilbert-Schmidt norm. Moreover it is also uniform with respect to the dimension $d$. Namely, there is a constant $K(M)$ only depending on $M$ such that if $\left(A^{\prime}, B^{\prime}, C^{\prime}, D^{\prime}\right) \in B_{3 / 2, M}^{d}$ and $(A, B, C, D) \in B_{3 / 2, M}^{d}$ are such that $\left\|A^{\prime}-A\right\| \leq \delta$, $\left\|B^{\prime}-B\right\| \leq \delta,\left\|C^{\prime}-C\right\| \leq \delta$ and $\left\|D^{\prime}-D\right\| \leq \delta$, then

$$
\left|\operatorname{Tr}\left(Q_{\eta_{0}}\right)\left(A^{\prime}, B^{\prime}, C^{\prime}, D^{\prime}\right)-\operatorname{Tr}\left(Q_{\eta_{0}}\right)(A, B, C, D)\right| \leq K(M) \delta .
$$

From all this, we deduce that, for small enough $\delta$,

$$
\operatorname{Tr}\left(Q_{\eta_{0}}(A, B, C, D)\right) \geq \operatorname{Tr}\left(Q_{\eta_{0}}\left(A^{\prime}, B^{\prime}, C^{\prime}, D^{\prime}\right)\right)-m / 2 .
$$

Since $A^{\prime}, B^{\prime}, C^{\prime}, D^{\prime}$ commute, we have $\operatorname{Tr}\left(Q_{\eta_{0}}\left(A^{\prime}, B^{\prime}, C^{\prime}, D^{\prime}\right)\right) \geq m$. We then get

$$
\operatorname{Tr}\left(Q_{\eta_{0}}(A, B, C, D)\right) \geq m / 2,
$$

a contradiction. 
We have shown the existence of some $\epsilon>0$ such that for all $d$ and $(A, B, C, D) \in$ $S_{1, M}^{d}$, we have

$$
\operatorname{Tr}\left(Q_{\eta_{0}}(A, B, C, D)\right) \geq \epsilon .
$$

Hence, $\operatorname{Tr}\left(Q_{\eta_{0}-\epsilon}(A, B, C, D)\right) \geq 0$ for all quadruple $(A, B, C, D)$ of symmetric matrices whose operator norm is bounded by $M$.

\section{REFERENCES}

[Ar] Emil Artin, Über die Zerlegung definiter Funktionen in Quadrate (German), Abh. Math. Sem. Univ. Hamburg 5 (1927), no. 1, 100-115, DOI 10.1007/BF02952513. MR3069468

[Bu] S. Burgdorf, Trace-positive polynomials, sums of hermitian squares and the tracial moment problem, http://nbn-resolving.de/urn:nbn:de:bsz:352-139805, thesis 2011.

[BK] Sabine Burgdorf and Igor Klep, Trace-positive polynomials and the quartic tracial moment problem (English, with English and French summaries), C. R. Math. Acad. Sci. Paris 348 (2010), no. 13-14, 721-726, DOI 10.1016/j.crma.2010.06.005. MR2671148 (2011j:16040)

[CKP] K. Cafuta, I. Klep, J. Povh, NCSOStools: A computer algebra system for symbolic and numerical computation with noncommutative polynomials, http://ncsostools.fis.unm.si.

[CL] Man Duen Choi and Tsit Yuen Lam, An old question of Hilbert, Conference on Quadratic Forms - 1976 (Proc. Conf., Queen's Univ., Kingston, Ont., 1976), Queen's Univ., Kingston, Ont., 1977, pp. 385-405. Queen's Papers in Pure and Appl. Math., No. 46. MR.0498375 (58 \#16503)

[Gy] L. Glebsky, Almost commuting matrices with respect to normalized Hilbert-Schmidt norm., preprint arXiv 1002.3082v1 (2010).

[Hi] David Hilbert, Ueber die Darstellung definiter Formen als Summe von Formenquadraten (German), Math. Ann. 32 (1888), no. 3, 342-350, DOI 10.1007/BF01443605. MR.1510517

[He] J. William Helton, "Positive" noncommutative polynomials are sums of squares, Ann. of Math. (2) 156 (2002), no. 2, 675-694, DOI 10.2307/3597203. MR.1933721 (2003k:12002)

[KS] Igor Klep and Markus Schweighofer, Connes' embedding conjecture and sums of Hermitian squares, Adv. Math. 217 (2008), no. 4, 1816-1837, DOI 10.1016/j.aim.2007.09.016. MR:2382741 (2009g:46109)

[Ln] Huaxin Lin, Approximation by normal elements with finite spectra in $C^{*}$-algebras of real rank zero, Pacific J. Math. 173 (1996), no. 2, 443-489. MR1394400 (98h:46059)

[Rw] Louis Halle Rowen, Polynomial identities in ring theory, Pure and Applied Mathematics, vol. 84, Academic Press Inc. [Harcourt Brace Jovanovich Publishers], New York, 1980. MR.576061(82a:16021)

[Mo] T. S. Motzkin, The arithmetic-geometric inequality, Inequalities (Proc. Sympos. WrightPatterson Air Force Base, Ohio, 1965), Academic Press, New York, 1967, pp. 205-224. MR0223521 (36 \#6569)

[Ro] Raphael M. Robinson, Some definite polynomials which are not sums of squares of real polynomials, Selected questions of algebra and logic (collection dedicated to the memory of A. I. Mal'cev) (Russian), Izdat. "Nauka" Sibirsk. Otdel., Novosibirsk, 1973, pp. 264-282. MR.0337878 (49 \#2647)

[Ve] Gregory C. Verchota, Noncoercive sums of squares in $\mathbb{R}\left[x_{1}, \ldots, x_{n}\right]$, J. Pure Appl. Algebra 214 (2010), no. 3, 236-250, DOI 10.1016/j.jpaa.2009.05.012. MR2559694 (2011b:12003)

IRmar (CNRS, URA 305), Université de Rennes 1, Campus de Beaulieu, 35042 Rennes Cedex, France

E-mail address: ronan.quarez@univ-rennes1.fr 